\title{
Algunas razones y elementos para hacer una reforma pensional en Colombia*
}

\section{Some reasons and elements for reforming the pension system in Colombia}

\section{\{ Leonardo Cañón Ortegón $\}^{\text {** }}$}

Recibido: Io de enero de 2020. Aprobado: 30 de julio de 2020. Dor: https://doi.org/IO.I860I/25390406. $\mathrm{n} 5.02$

Abogado del Colegio Mayor de Nuestra Señora del Rosario, máster en Derecho Social y en Gobierno y Administración Pública de la Universidad de Lovaina, Bélgica, exviceministro de Trabajo y Seguridad Social, profesor de seguridad social en la Universidades Externado, Rosario y Javeriana, autor de libros y artículos sobre seguridad social, salud, subsidio familiar, etc., asesor y consultor de organizaciones nacionales e internacionales de seguridad social y salud. 


\section{RESUMEN}

En Colombia se debe hacer una reforma integral en materia pensional para dar protección a la población mayor, en la cual se aborden múltiples razones expuestas por diversos sectores de la sociedad y se tengan en cuenta elementos relacionados con la estructura demográfica de la población, la nueva composición del mercado de trabajo y una evaluación del actual Sistema General de Pensiones, a partir de los parámetros establecidos por la Constitución Política de I99ı, de manera que se revisen los ajustes que se han hecho al actual sistema, se haga una reflexión profunda sobre el contenido del derecho a las pensiones de invalidez, vejez y de sobrevivencia, sin que se limite al reconocimiento de las mesadas pensionales, dejando de lado las necesidades asistenciales de salud, hospitalización y demás servicios sociales complementarios que requiere la población mayor; los mecanismos establecidos para mantener el poder adquisitivo de las pensiones; se replantee la eficacia de las indemnizaciones sustitutivas y las devoluciones de saldos, frente a la garantía del derecho pensional de las personas; se adopte una política integral de pensiones que pueda ser ejecutada al más alto nivel del Estado y se establezcan mecanismos financieros permanentes que garanticen la sostenibilidad financiera del sistema pensional en Colombia, mediante una metodología que incorpore esquemas de tipo contributivo y distributivo de los recursos para que el Estado pueda garantizar los derecho irrenunciables a la seguridad social y a la protección social en condiciones de igualdad para todos los habitantes.

\section{Palabras clave}

Reforma integral, estructura demográfica, mercado de trabajo, progresividad, igualdad, población mayor, política social, igualdad, sistema general de pensiones, sostenibilidad financiera, derecho irrenunciable, seguridad social, protección social.

\section{Abstract}

To protect the elderly population, the Colombian Government needs to rethink the current pension system, considering the issues pointed by different sectors of Colombian society, such as the diverse demographic dynamics, the new composition of the labor market, and the parameters established by the i99i Political Constitution. This reform must guarantee people's access to retirement, disability, and survival pensions, health care needs, hospitalization, and other complementary social services that the elderly population requires. Therefore, it has to review the mechanisms established to keep the purchasing power of 
pensions and rethink the effectiveness of replacement indemnities and repayments of balances to guarantee people's pension rights. Likewise, the Government must adopt a comprehensive public policy, executed at the highest level of the State, which allows establishing permanent financial mechanisms to guarantee the economic sustainability of the pension system in Colombia. This reform must implement a mixed scheme to ensure resources that enable the Government to secure the inalienable rights to social security and social protection under conditions of equality for all inhabitants.

\section{KEYWORDS}

Integral reform, demographic structure, work market, progressiveness, equality, older population, social policy, equality, general pension system, financial sustainability, inalienable right, social security, social protection.

\section{INTRODUCCIÓN}

Cumplidos cinco lustros desde cuando se creó en Colombia el Sistema General de Pensiones, como parte integrante del Sistema de Seguridad Social Integral, mediante la expedición de la Ley ioo de 1993 y los ajustes importantes adoptados con la expedición de las Leyes 860 de 2002 y 797 de 2003 y, especialmente, con la expedición del Acto Legislativo n..$^{\circ}$ 5 de 2005, es válido que la sociedad colombiana reflexione a fondo sobre lo que puede ser una reforma integral en materia pensional a partir de una evaluación de la situación sociodemográfica actual de la población mayor, de las características actuales del mercado de trabajo y la situación económica de la población mayor de 60 años, así como la evaluación objetiva al Sistema General de Pensiones, que entró en vigencia a partir de abril de 1994, conforme a los parámetros establecidos por la Constitución Política de I99I, en la que se consagraron los derechos a la seguridad social, a la salud y a la protección social para todas las personas y, en especial, para los denominados grupos vulnerables, como los niños, las personas de tercera edad o las personas en situación de discapacidad. De igual forma, en esta Carta Política se declararon las actividades de seguridad social como servicios públicos de carácter obligatorio, que deben ser garantizados por el Estado, con sujeción a los principios de solidaridad, universalidad y eficiencia ${ }^{ }$.

I Constitución Política de Colombia, art. 48 . 


\section{Algunas razones para hacer una Reforma Pensional}

Las razones que diversos sectores de la sociedad colombiana han dado para realizar una reforma al sistema pensional, apuntan mayoritariamente a simples ajustes paramétricos para aumentar la edad de pensión de vejez, el incremento del tiempo de aportes o cotizaciones, la igualdad de tratamiento entre hombres y mujeres; en cuanto a la edad exigida para obtener el derecho a la pensión de vejez, la disminución de la tasa de reemplazo, las limitaciones a los alcances de las pensiones de sobrevivencia, la unificación de los regímenes generales, las modificaciones en la organización administrativa, de vigilancia y control del vigente Sistema General de Pensiones, hasta la adopción de una política pública de protección social para la población mayor que no se limite conceptualmente al derecho a garantizar un ingreso monetario, representado en una renta básica o un ingreso solidario, sino que también incluya la protección asistencial y social que demandan las necesidades y limitaciones propias de la población mayor en áreas como la prestación de los servicios médicos, hospitalarios, farmacéuticos, de rehabilitación, de asistencia social, así como el suministro de otros bienes o artículos y servicios sociales complementarios.

También se mencionan con insistencia los problemas de financiación de los actuales regímenes generales; el costo fiscal que el erario asume para atender los pagos de las pensiones actuales; las bajas coberturas en materia de afiliación y pago de cotizaciones al Sistema General de Pensiones y de personas pensionadas; las inequidades existentes dentro de los regímenes generales actuales y en relación con los regímenes de excepción y especiales que aún subsisten; la baja propensión de ahorro para financiar los requerimientos futuros de la población mayor; la falta de educación e información financiera para la construcción de mecanismos de protección durante la vejez, y los resultados que empiezan a darse en los regímenes generales sobre el número de pensionados con respecto al total de la población mayor, los montos de las pensiones o de los subsidios provenientes de mecanismos complementarios al sistema general de pensiones y sobre los volúmenes de personas que están recibiendo indemnizaciones sustitutivas o devoluciones de saldos, en reemplazo del propio ${ }^{2}$.

De igual manera, se mencionan los altos niveles de informalidad en la población trabajadora, los bajos niveles en los aportes para pensiones y la baja densidad de las cotizaciones, derivados de la informalidad, el desempleo, la siones en Colombia (Bogotá: 2017), 96 y ss. https://www.contraloria.gov.co/documents/2018I/452 I24/ Análisis+y+discusión+técnica+de+la+situación+del+Sistema+General+de+Pensiones+-Dic+20I 7. pdf/oeoedd $8 c-4$ bf I-40Ic-aea I-fo4e 36 facdd 7 ? version=I.o 
movilidad laboral y la falta de educación financiera para la vejez, así como la situación de pobreza de gran parte de la población mayor ${ }^{3}$.

\section{Algunos elementos para tener en CUenta en UnA Reforma Pensional}

Sin perjuicio de profundizar en el señalamiento de razones que se están esgrimiendo en los diversos sectores y componentes de la sociedad colombiana para impulsar una reforma pensional integral, a continuación, presento algunos de los elementos que deberían tenerse en cuenta para el estudio, la discusión y el eventual trámite de una reforma pensional en el Congreso de la Republica de Colombia.

\section{LOS CAMBIOS EN LA ESTRUCTURA DEMOGRÁFICA DE LA POBLACIÓN COLOMBIANA}

Además de reconocer los avances que ha tenido la sociedad colombiana en la disminución de las tasas de fecundidad y natalidad durante las últimas décadas es preciso reconocer los avances que se han venido dando en el incremento de la esperanza de vida. Diversos estudios dan cuenta de que, en la actualidad, según el DANE, el I3,4\% de la población colombiana, o sea, alrededor de 6,5 millones de personas, es mayor de sesenta años ${ }^{4}$, de los cuales solo un poco más de 2300000 cuentan con una pensión y que para el año 2050 , el país tendrá alrededor de 14,5 millones de personas mayores, de los cuales muy pocos recibirán pensión con el actual sistema ${ }^{5}$.

Existen estudios, análisis y planteamientos confiables sobre el incremento de las necesidades propias de la población en cuanto a las necesidades en materia de servicios médicos, hospitalarios, farmacéuticos, de ayuda, mantenimiento, rehabilitación, vivienda, centros de esparcimiento, alimentación y nutrición, entre otros, que será preciso atender con recursos provenientes de las pensiones de tipo contributivo o con esquemas no contributivos de financiación pública, resultantes de la solidaridad de la población con mayores recursos.

Los mecanismos de protección para la población mayor tendrán que atender a las necesidades identificadas a nivel nacional, regional y local, en las zonas urbanas y rurales, en la participación formal o informal en el mercado de trabajo, además de las originadas, entre otros factores, por las nuevas 
composiciones de las familias, los niveles educativos, económicos y culturales de la población.

\section{LAS CARACTERÍSTICAS ACTUALES DEL MERCADO DE TRABAJO}

A partir de la década de I990, y en razón del surgimiento de fenómenos como la apertura económica, la globalización de la economía, la modernización del Estado, la privatización, las necesidades de flexibilización de la contratación, la incorporación de nuevas tecnologías al mercado de trabajo, la mayor participación de la mujer en el sistema educativo y su incorporación al mercado de trabajo, entre otros, que han dado lugar a la expedición de reformas laborales como las contenidas en la Ley 50 de 1990 y la Ley 789 de 2002 , han surgido nuevas formas de trabajo, ocupaciones y actividades productivas, con relaciones distintas a las de dependencia o subordinación, que generan la protección patronal prevista en el artículo 9. ${ }^{\circ}$ del Código Sustantivo del Trabajo, cuya actualización ha debido hacerse para dar protección al trabajo "en todas sus modalidades", garantizando condiciones dignas y justas, como lo determinó la Constitución Política de $199 \mathrm{I}^{[6]}$. En ese sentido, se ha dado un creciente desarrollo del trabajo informal, que representa alrededor del 60\% de la población ocupada y que se manifiesta en nuevas ocupaciones o formas de trabajo como freelancers; cuidadores de inválidos, enfermos o personas mayores; trabajadores autónomos; emprendedores; rapitenderos; trabajadores independientes; trabajadores paramédicos; deportistas; trabajadores de cosecha; vendedores ambulantes; estilistas; domiciliarios; trabajadores en casa, entre otras ocupaciones que, aunque aportan a la productividad de la economía del país, no se han "formalizado" y tampoco participan del Sistema de Seguridad Social Integral, menos aún del Sistema General de Pensiones, pese a los esfuerzos realizados por parte de los últimos gobiernos en materia de "formalización y generación de empleo"

En términos reales, para estos grupos poblacionales no es atractivo ingresar al sistema de protección laboral regulado por el Código Sustantivo del Trabajo para los trabajadores dependientes, como tampoco al Sistema de Seguridad Social Integral y, al interior de este, al Sistema General de Pensiones, fundamentalmente por los altos costos que ello les representa. Menos atractivo resulta hacerlo por medio de un empleador o de un contratante de prestación de servicios.

6 Const., art. 25 .

7 Ley r429 del 29 de diciembre, sobre formalización y generación de empleo, Diario Oficial n. ${ }^{\circ}$ 47.937. http://www.secretariasenado.gov.co/senado/basedoc/ley_I429_20Io.html 
Valdría la pena, entonces, replantear el concepto de formalización, sin limitarlo al establecimiento de una relación laboral con contratos de trabajo previstos en la legislación vigente. Esto es algo que aparentemente los empleadores no quieren realizar por los efectos sobre los costos de las nóminas, a lo que los trabajadores independientes se rehúsan, por tener que asumir los costos de las cotizaciones a los regímenes de pensiones (I $6 \%$ ), de salud (I $2,5 \%$ ), de riesgos laborales, según la clase y grado de riesgo de las actividades, y los denominados parafiscales para financiar los programas del SENA o del ICBF y del subsidio familiar que administran las Cajas de Compensación. Además, frente a estos, el propio Estado desestimula con las nóminas paralelas y los procesos de tercerización de muchos de los servicios y actividades que le corresponde desarrollar.

Para tratar de resolver el problema de la informalidad laboral y sus efectos sobre la vinculación de la población ocupada en la seguridad social habrá que hacer un gran esfuerzo para actualizar el Estatuto del Trabajo, expedir regulaciones para garantizar el derecho al trabajo, digno y justo, en las diversas modalidades de trabajo, como ha tratado de hacerse con el servicio doméstico, el teletrabajo y el trabajo remoto, los trabajadores por horas o los trabajadores con contratos de duración inferior a un mes, reconociendo las características propias y particulares de estas formas de trabajar a fin de lograr su incorporación a la seguridad social en salud, pensiones, riesgos laborales, protección familiar.

Este gran esfuerzo de formalización laboral deberá comprender estrategias para estimular el ahorro y los aportes para la protección de la vejez por parte de la población que aporta a la productividad del país desde la informalidad, estableciendo un sistema atractivo que los incorpore a la seguridad social y, por tanto, garantizando que se hagan aportes para financiar las pensiones de tipo contributivo, de corte obligatorio o voluntario. En ese sentido, no es razonable seguir estimulando el ahorro privado entre las personas que ni siquiera disponen de recursos suficientes para garantizar una subsistencia digna, como sucede con los Servicios Sociales Complementarios Beneficios Económicos Periódicos (BEPs).

\section{Una evaluación objetiva del actual Sistema General de Pensiones}

Por lo expuesto en el anterior apartado, es necesario realizar una evaluación objetiva del actual Sistema General de Pensiones en aspectos fundamentales como la cobertura poblacional, tanto de los afiliados como de los cotizantes activos e inactivos; de los pensionados por vejez, invalidez y sobrevivientes y de los retos existentes en materia de cobertura de la población mayor del país, para 
conjurar los denominados "cuatro males de las jubilaciones": la informalidad, el déficit de financiación, la inequidad, los privilegios y la evasión ${ }^{8}$.

Esta evaluación debe incluir la eficiencia y eficacia de los mecanismos complementarios al Sistema General de Pensiones, como los beneficios económicos periódicos (вEPs), los subsidios a las cotizaciones para pensiones, los subsidios a las pensiones altas y a las de salario mínimo, los subsidios del programa Colombia Mayor, los subsidios para la cuenta de subsistencia y del Fondo de Pensión Mínima del Régimen de Ahorro Individual con Solidaridad (RAIS), entre otros 9 , lo mismo que la función de las indemnizaciones sustitutivas o las devoluciones de saldos del Sistema General de Pensiones vigente u otros como el de la pensión familiar, establecidos en un intento de brindar protección económica mínima, subsidios alimentarios y de aportes a salud por sumas inferiores a los montos mínimos de pensión establecidos por la Carta Política, frente a la necesaria atención de las necesidades reales de la población mayor.

El Sistema de Seguridad Social Integral, creado en I993, ha tenido importantes avances en materia de cobertura en salud, logrando incluir a más del $90 \%$ de la población, a través de los regímenes contributivo y subsidiado, pero no ha ocurrido lo mismo en el campo de las pensiones, pues de aproximadamente I6 millones de afiliados, menos del $50 \%$ está activo. Tampoco ocurre esto en el sistema de riesgos laborales, en el que apenas se cuenta con poco más de Io millones de afiliados a las Administradoras de Riesgos Laborales (ARL).

Para el caso específico del Sistema General de Pensiones habrá que reflexionar y tomar decisiones en relación con los siguientes aspectos: una densidad de cotizaciones inferior al $50 \%$ y la falta de regularidad en la cotización derivada de la alta movilidad laboral para cumplir con los tiempos mínimos de aportes exigidos para tener derecho a una pensión en los dos regímenes generales, de I 300 y I I 50 semanas de cotización; la escasa eficacia de los Fondos de Solidaridad Pensional para subsidiar cotizaciones a las personas de menores recursos para alcanzar niveles de pensión importantes y del Fondo de Pensión de Garantía de Pensión mínima del rais; las actuales tendencias de la población a buscar el traslado de un régimen a otro, por los resultados en cuanto a monto y cantidad de pensionados, tanto en el Régimen de Prima Media (R PM) como en el raIs, y las crecientes cifras de indemnizaciones sustitutivas y de devoluciones de saldos que se están produciendo, quedando, en la práctica, sin protección efectiva a la población mayor. com/economia/sectores/males-de-las-pensiones-en-colombia-25 I 28

$9 \quad$ "Mientras hay una reforma, estas son las opciones". El Tiempo, I3 de agosto de 20 I8. 
Mención especial habrá de hacerse sobre las alertas en torno a la sostenibilidad del Sistema General de Pensiones con recursos provenientes del presupuesto nacional, especialmente aquellos derivados de los compromisos adquiridos por la Nación, provenientes de los regímenes de solidaridad intergeneracional, asumidos por las entidades públicas con anterioridad a las reformas por medio del FOPEP, la UGPP, ISS ARL, ISS trabajadores, el Ministerio de Obras Públicas, intra, prosocial, entre otros organismos e instituciones, los regímenes exceptuados y especiales, como los de las Fuerzas Militares, el Magisterio, los de los congresistas, las Altas Cortes, el régimen de transición y los de los Seguros Sociales Obligatorios, la mayoría de los cuales están siendo administrados por Colpensiones, FOPEP, las cajas y los fondos de previsión social $^{\mathrm{ro}}$.

En este análisis tendrá que considerarse la viabilidad financiera de los dos regímenes generales, sobre todo si la reforma debe circunscribirse únicamente a la modificación de los dos regímenes generales o si se incorporan en ella las reformas a los demás regímenes de pensiones, de excepción o especiales. De igual forma, tendrá que definirse si la reforma debe limitarse a las pensiones de vejez o si también deben ajustarse la financiación, cobertura y demás características de las pensiones de invalidez y de sobrevivencia. Asimismo, es importante establecer si esta debería abarcar, o no, los servicios asistenciales de salud, farmacéuticos, de rehabilitación, asistencia social y demás servicios sociales complementarios, según las características y necesidades de la población mayor.

\section{DESARROLLO dE LOS PARÁMETROS CONSTITUCIONALES SOBRE PROTECCIÓN A LA POBLACIÓN MAYOR}

En la Constitución Política de r99i se establecieron las bases que sirven de fundamento político y jurídico para el desarrollo y la implementación de la seguridad social, la salud y la protección social, en general, específicamente para garantizar los derechos a la seguridad social, la salud y protección social para todas las personas. Lo anterior se concreta en el artículo 46, cuyo texto asegura la protección y asistencia a las personas de la tercera edad, que deben ser asumidas como parámetros constitucionales que orienten la reforma pensional integral, enfocados en aspectos como los siguientes:

I. Los fundamentos del Estado social de derecho, consagrados en el artículo r. ${ }^{\circ}$ de la Constitución, aplicables todos con la reforma pensional 
integral tendrán que sustentar cualquier política de protección social de la población mayor, a saber: el respeto a la dignidad humana de todas las personas; el trabajo formal o informal en todas sus modalidades; la solidaridad social como principio fundamental de la seguridad y la protección social, y la prevalencia del interés general sobre el interés particular, como característica esencial de los servicios públicos de la seguridad social, de la salud y la protección social, que corresponde al Estado colombiano garantizar a toda la población.

2. La aplicación de los principios que inspiran la seguridad social, la salud y la protección social, establecidos por la propia Constitución, la legislación vigente y las múltiples decisiones jurisprudenciales sobre la materia, como son la solidaridad, la universalidad, la integralidad, la eficiencia, la continuidad o la progresividad, la continuidad o la sostenibilidad financiera.

3. La consagración de los derechos fundamentales a la seguridad social, la salud y la protección social para todos los habitantes, especialmente para quienes por sus condiciones físicas, económicas y mentales se encuentren en situación de debilidad manifiesta, sobre los cuales ya no existe discusión alguna en torno al carácter de derechos fundamentales autónomos, cuya garantía y materialización corresponde al Estado colombiano, con base en los artículos 48, 49, I3 y 46 de la Constitución Política y el gran desarrollo legislativo y jurisprudencial de los últimos veinticinco años de vigencia de los actuales Sistemas de Seguridad Social Integral, de Pensiones y de la Protección Social.

4. Las normas declarativas contenidas en la Constitución sobre los servicios públicos de carácter "obligatorio" y "a cargo del Estado", relacionados con las actividades de seguridad social, de salud y de la protección social, especialmente aquellos prestados a la población mayor, indicados en los artículos 48, 49 y 46 de la Carta Política, así como en sus desarrollos legislativos y jurisprudenciales para garantizar los "servicios de seguridad social integral" de las personas de tercera edad y promover su integración a la vida activa y comunitaria, como responsabilidad del Estado, la sociedad y la familia.

5. El deber de solidaridad social establecido por la Constitución establecida para que todos los miembros de la comunidad nacional puedan cumplir con su obligación individual y colectiva de responder "con acciones humanitarias en situaciones que pongan en peligro la vida o la salud de las personas", como lo establece el artículo 95, numeral 2 de la Constitución. 
6. La incorporación a la Constitución Política del compromiso que tiene el Estado colombiano de garantizar los derechos pensionales, respetar los derechos adquiridos y la sostenibilidad financiera para el sistema general de pensiones, mediante el Acto Legislativo n. ${ }^{\circ}$ or de 2005.

\section{LA NECESIDAD DE UNA POLÍTICA PÚBLICA DE PROTECCIÓN INTEGRAL DE LA POBLACIÓN MAYOR}

La necesidad de establecer una política pública de protección a la población mayor es un elemento que se deriva de la obligación que tienen las autoridades de proteger la vida, la honra y los bienes de todas las personas residentes en Colombia y de asegurar el cumplimiento de los deberes sociales del Estado. Esta política no debe estar limitada al reconocimiento de un ingreso monetario, derivado del nivel de ahorro que cada individuo pueda llegar a acumular en su vida activa, sino que también debe incluir otras prestaciones de carácter indemnizatorio, además de las manifestaciones de solidaridad por parte de la sociedad, en general, y la familia, en particular, para atender las necesidades de las personas de la tercera edad.

Antes de la Ley roo de I993, por ejemplo, el derecho a las pensiones de jubilación o de vejez comprendía la mesada pensional que se paga en dinero, los servicios médicos, quirúrgicos, hospitalarios, farmacéuticos y de rehabilitación, con un aporte de tan solo el 5 \% del valor de la mesada en razón de la mayor utilización de estos ${ }^{\mathrm{II}}$, y los servicios sociales complementarios que se prestaban a los pensionados del Seguro Social por parte del Fondo de Servicios Sociales Complementarios, que se otorgaban directamente por el Instituto de Seguros Sociales, las cajas de previsión social y en convenios con las cajas de compensación familiar ${ }^{\mathrm{I2}}$.

La política de protección a la vejez debe ser una política pública del Estado colombiano que desarrolle un concepto de protección social, implementando una pensión que no esté limitada al ahorro y a la satisfacción de necesidades de ingreso, sino también atienda otras necesidades propias de la situación de vejez, invalidez, orfandad, sobrevivencia y dependencia económica, ubicada en los altos niveles del Estado y que involucre a todas las entidades nacionales, departamentales, distritales y municipales, con nuevas fuentes de financiación,

Ley 12 del 16 de enero de 1975 . Sobre el régimen de pensiones de jubilación, Diario Oficial $\mathrm{n}{ }^{\circ}$ 34.245 , art. 7 . 
pero que involucre a diferentes sectores y se desarrolle mediante un manejo económico integral.

Con la legislación de I993, sus modificaciones y desarrollos, se produjo una clara violación del principio de progresividad y prohibición de regresividad, consagrado en la Constitución y desarrollado por la jurisprudencia ${ }^{13}$, por cuanto el derecho pensional se limitó a las mesadas pensionales resultantes del ahorro individual durante la vida activa y se estableció que para tener acceso a la cobertura en salud, sin derecho a las prestaciones económicas del régimen común, es necesario que el pensionado se afilie al Sistema General de Seguridad Social en Salud y cotice el i2 \% del valor de su pensión.

Los beneficios del Fondo de Servicios Sociales Complementarios de los seguros sociales obligatorios desaparecieron y, con ellos, la posibilidad de participar de algunos servicios o programas sociales que prestan las cajas de compensación familiar, en materia cultural y de recreación. Para tener acceso a los programas para adultos mayores, estos deben afiliarse como pensionados y hacer los aportes correspondientes del o,6\% o del $2 \%$ del valor de su mesada pensional ${ }^{14}$, lo que constituye un franco retroceso en lo que respecta a la protección de la población mayor en Colombia, que debe ser revisado sin dilaciones.

La construcción y definición de esa política social debe estar sustentada en las necesidades de la población, sus características sociodemográficas y el establecimiento de mecanismos como la definición de un concepto de pensión que no esté limitado a los aspectos monetarios, sino que se complemente con toda la protección, a través de servicios asistenciales de salud, como la prevención de enfermedades; la promoción de la salud y la protección médica, hospitalaria y de rehabilitación; los servicios de diagnóstico, tratamiento y de paliación de las enfermedades, y demás servicios sociales complementarios de mantenimiento, ayuda y bienestar que se requieran.

Dentro de esta política deben establecerse los permanentes esfuerzos que el Estado y la sociedad y la familia deben realizar, en términos económicos, presupuestales, administrativos, organizacionales, de vigilancia y control para garantizar su adecuada aplicación tanto a nivel nacional como regional y local, sin discriminaciones de cualquier naturaleza. Así, esta no debe ser responsabilidad exclusiva del Ministerio de Salud y Protección social, del Ministerio de tucional. 
Trabajo o de la Comisión Intersectorial de Pensiones y Beneficios Económicos Periódicos, que señala las responsabilidades del Ministerio de Trabajo ${ }^{15}$.

Esta política debe incorporar mecanismos complementarios como los BEPs, el programa denominado Colombia Mayor, el Fondo de Solidaridad Pensional y Subsistencia, en su función de subsidiar las cotizaciones a pensiones y completar las pensiones de subsistencia, lo mismo que la del Fondo de Garantía de Pensión Mínima del rais.

Dentro de esta debe incluirse un componente que regule las pensiones de invalidez, tanto del Régimen Común como del Régimen de Riesgos Laborales, para eliminar la inequidad existente entre los dos regímenes generales vigentes, como ocurre en materia del reconocimiento de los porcentajes de la incapacidad temporal y la indemnización por incapacidad permanentemente parcial, en su contenido, alcances y financiación.

Esta política debe proponer o por lo menos contemplar nuevas fuentes posibles de financiación, de manera similar a lo que ocurre en el Sistema General de Seguridad Social en Salud, con base en una estructura institucional que involucre, a nivel nacional, las entidades territoriales y locales para establecer los mecanismos necesarios que garanticen el equilibro financiero del Sistema General de Pensiones.

No es posible seguir tomando medidas ineficaces, como recaudar los ahorros de la población de niveles i, ir y iI del Sistema de Identificación de Beneficiarios en Salud, Sisbén, para financiar el servicio social complementario de los Beps. En cambio, sí podría recaudarse de los ingresos y recursos de los trabajadores independientes y autónomos, con capacidad de ahorro en el sector financiero o en el sector informal de la economía. Con esto, se evitarían hechos ocurridos como la transformación de la naturaleza jurídica inicial de Colpensiones, que pasó de ser una empresa Industrial y Comercial del Estado a una empresa financiera de carácter especial, porque el sector financiero no estuvo en condición de administrar las pequeñas cuentas de ahorro de los afiliados al BEPs, dados los costos que implicaba el recaudo de los exiguos ahorros, por lo que el Estado tuvo que acudir a cerca de 30 ooo instituciones o agencias de recaudo de los ahorros de los afiliados al programa de beneficios periódicos.

En otras palabras, no es viable seguir buscando recursos para atender a la población mayor en el ahorro de los más pobres, como si estos gozaran de las mismas condiciones de las que goza la población activa y la población ocupada, que también aporta a la productividad del país de manera informal, como viene ocurriendo en el caso del Sistema General de Seguridad Social en Salud. 


\section{LO QUE NO DEBE haCerSe EN MATERIA DE REFORMA PENSIONAL}

Para hacer énfasis en la necesidad de hacer una reforma pensional integral que responda a las nuevas realidades de protección social de la población mayor, es necesario relacionar en materia pensional algunos aspectos que han afectado puntualmente la cobertura de los riesgos derivados por vejez, invalidez y muerte en el Sistema General de Pensiones:

a) El contenido y los alcances de algunos servicios como los asistenciales, hospitalarios y farmacéuticos, que antes de la Ley roo de 1993 formaban parte del derecho a las pensiones y que se vieron reducidos por el concepto de pensión al ingreso monetario que se otorga en forma de las mesadas, violando el principio de progresividad y de prohibición de regresividad y obligando al pensionado a afiliarse al Sistema General de Seguridad Social en salud con una cotización que inició siendo del $\mathrm{I} 2,5 \%$, igual a la de los afiliados al régimen contributivo en salud y que luego se estableció en el I $2 \%$, pese a los intentos por disminuir la cotización al $4 \%$ del valor de las mesadas pensionales. Esta reducción también violó el principio de la igualdad, por restringir la cobertura de los pensionados en el régimen de enfermedad común a la cobertura prestacional, sin derecho a prestaciones económicas del régimen de aseguramiento en salud en caso de incapacidad temporal, incapacidad permanente parcial, invalidez, con una clara desigualdad de tratamiento del derecho a la salud con los demás afiliados al régimen de enfermedad común, con el mismo nivel de financiación del io \% de la cotización.

b) El tratamiento discriminatorio que se da al incremento anual de las mesadas pensionales para mantener el poder adquisitivo constante, estableciendo que los pensionados con pensiones equivalentes a un salario mínimo legal mensual reciban incrementos equivalentes al valor del incremento del salario mínimo y el incremento del valor del IPC para los demás pensionados, violando el principio de igualdad consagrado en la Constitución y el mandato según el cual "la ley definirá los medios para que los recursos destinados a pensiones mantengan su poder adquisitivo constante" ${ }^{\text {.6 }}$.

c) Los conceptos de indemnización sustitutiva y devolución de saldos que, en aplicación del criterio del derecho individual para recuperar los ahorros destinados a financiar la pensión durante la vida productiva, 
resultan en contravía de la aplicación del principio de solidaridad de la seguridad social consagrado en la Constitución y progresivamente dejan sin cobertura pensional a los grandes riesgos de vejez, invalidez y sobrevivencia de los colombianos.

d) Las medidas que se tomaron de manera transitoria, con ocasión de la pandemia de la covid-ı9, que afectaron de manera grave el derecho a la seguridad social en materia pensional. Ejemplo de esto fue la expedición del Decreto 558 de 2020 , tramitado con base en las facultades del estado de emergencia económica social y ecológica, establecida por el Decreto 4I7 de 2020, para permitir que durante los meses de abril y mayo del 2020 , solo se hicieran aportes al sistema de pensiones del $3 \%$ para financiar los seguros previsiones de invalidez y muerte, dejando desprotegidos a los trabajadores en materia de cobertura de los riesgos asociados al sistema de pensiones ${ }^{17}$.

Dicha disposición fue declarada inexequible por parte de la Corte Constitucional mediante Sentencia C-258 de 2020, pues en el ejercicio de las facultades de excepción establecidas en el artículo 215 de la Constitución está claro que no se pueden afectar derechos sociales de los trabajadores, así la propia sentencia haya ordenado al Gobierno establecer los mecanismos para recaudar que los aportes no pagados por aportes al Sistema General de Pensiones por parte de los empleadores y contratantes.

Esta decisión ha traído como consecuencia el cese de aportes al Sistema General de Pensiones, por parte de los afiliados activos, afectando seriamente el derecho a la protección contra los riesgos de vejez, invalidez y muerte de los afiliados en casos como los siguientes:

I. Las personas que fueron despedidas o terminaron su afiliación al Sistema General de Pensiones, vieron afectado su derecho a pensión, por falta de las semanas que dejaron de cotizarse.

2. Los recursos correspondientes a esas semanas de cotización, cercanos a los 2,8 billones de pesos, dejaron de concurrir al fondo público de Colpensiones o las cuentas individuales de las AFP, afectando el capital y los rendimientos e intereses de los afiliados.

3. Se generaron dificultades para el pago de los aportes no realizados por los empleadores, especialmente en los casos en que se terminó la relación laboral existente durante la vigencia de esta medida. pensiones, art. I. https://www.funcionpublica.gov.co/eva/gestornormativo/norma.php?i=I I 3620 
4. Muchas personas vieron afectado el reconocimiento de las pensiones de vejez, invalidez y de sobrevivencia, como la indemnización sustitutiva y la devolución de saldos, por falta de los recursos que se dejaron de recibir durante la vigencia de la medida.

Hechos que además podrían implicar:

5. La responsabilidad de los funcionarios del Estado que decretaron la emergencia económica social y ecológica, como las medidas extraordinarias decretadas para suspender el pago de cotizaciones al Sistema de Pensiones y diferir el pago de las sumas dejadas de pagar, por haber afectado el derecho fundamental a la seguridad social en pensiones de los afiliados al sistema.

6. La posible sanción a los funcionarios que tomaron dichas medidas, quienes podrían hacerse acreedores de medidas disciplinarias, según lo dispuesto en la Ley Estatutaria I37 de 1994, que regula el ejercicio de los estados de Excepción

7. La eventual responsabilidad del Estado por los perjuicios causados, derivados de los actos y hechos de los funcionarios que intervinieron en la toma de las medidas indicadas, cuyas dimensiones pueden resultar cuantiosas por el trascurrir del tiempo y por afectar a todos los afiliados al Sistema General de Pensiones, a pesar de que el Gobierno reglamente la forma de reponer esos aportes al Sistema de General de Pensiones en tres años y sin intereses, como lo dispuso la Corte Constitucional ${ }^{18}$.

\section{ANÁlisiS dE LAS PROPUESTAS FORMULADAS}

En Colombia se debe formular una propuesta adecuada y auténtica que, en lo posible, responda a las condiciones y necesidades de la población colombiana, y que sea el desarrollo de una política integral de protección a la población mayor. Los responsables de la elaboración técnica del proyecto, que sean puestos a consideración del legislador, deben tener en cuenta las diversas posibilidades que brindan la solidaridad general y la responsabilidad social para garantizar una pensión social o mínima de subsistencia, alternativa a las pensiones de tipo contributivo, de carácter obligatorio o voluntario, para quienes participan del sector productivo, a manera de los sistemas de pilares aplicados en otros 
países, que han planteado los análisis de la ocDe, del Ministerio de Trabajo y algunos gremios.

\section{¿Cómo Llevar a cabo La Reforma?}

Antes de configurar un proyecto de ley que, de ser aprobado debería convertirse en ley estatutaria, por tratarse de la regulación de derechos humanos y fundamentales de todas las personas, la propuesta podría ser construida por un equipo multidisciplinario, multisectorial, conformado por representantes del Gobierno, empleadores, trabajadores, pensionados, la academia, entre otros actores, $y$ fundamentarse sobre los siguientes ejes:

- Acoger, en forma simultánea, una reforma al estatuto del trabajo, para regular el trabajo en todas sus modalidades y en condiciones dignas y justas, de todas aquellas personas que laboran en formas diferentes a las relaciones laborales que constituyen empleadores y trabajadores dependientes, sin violar su derecho a la seguridad social y la protección social, y dando prioridad a la protección de la población mayor.

- Empezar por definir el campo de acción del sistema pensional colombiano, para dar protección mayor, que no esté limitado al reconocimiento de un ingreso monetario, sino que incluya el acceso oportuno, eficiente y adecuado a los servicios de salud y a los servicios sociales complementarios, requeridos para atender las necesidades de la población mayor colombiana.

- Emprender la construcción de una política integral de Estado sobre la protección social para la población mayor, que desarrolle los principios constitucionales en materia de seguridad social, salud y protección social.

- Incorporar como responsabilidad del Estado la definición, desarrollo y ejecución de la política de protección social para la población en un nivel alto de la estructura del Estado.

- Establecer un mecanismo de control permanente para garantizar la sostenibilidad financiera y, con ello, la financiación y estabilidad del sistema de protección social para la población mayor, como las leyes de programación y financiamiento de la seguridad social que se expide periódicamente para la seguridad social en Francia ${ }^{19}$. 
Finalmente, es importante advertir a los diferentes sectores de la comunidad, políticos y académicos, interesados en los temas sociales, que este artículo no tiene otro propósito distinto al de advertir las principales razones y elementos, a partir de los cuales, el país pudiera abordar el estudio, el análisis y la discusión de una reforma integral de la seguridad social y la protección social a fin de garantizar mejores niveles de bienestar y calidad de vida para la población colombiana mayor, en aplicación del principio de la solidaridad social que inspira al Estado social de derecho adoptado por la Constitución Política de i99ı.

\section{Bibliografía}

Asofondos. Al fin, ¿Cómo nos vamos a pensionar? El Tiempo, I3 de agosto 2018.

Contraloría General de la República. Análisis y discusión técnica de la situación del sistema general de pensiones de Colombia. Bogotá: 2017. https://www.contraloria.gov.co/documents/20I8I/452 I 24/Análisis+y+discusión+técnica+de+la+situación+del+Sistema+Gen eral+de+Pensiones+-Dic+20I7.pdf/oeoedd8c-4bf I-4o Ic-aea I-fo4e 36 facdd 7 ? version=I.o

Dane. Exposición de Juan Daniel Oviedo Arango. Bogotá: Universidad del Rosario, 2019.

El Tiempo. Los cuatro males de las jubilaciones. I3 de agosto de 2018 . https://www.eltiempo. com/economia/sectores/males-de-las-pensiones-en-colombia-25 I I 28

El Tiempo. Mientras hay una reforma, estas son las opciones. I3 de agosto de 2018.

Morvan, Patrick. Droit de la Protection Sociale, 8. ${ }^{a}$ ed. París: LexisNexis, 2017.

\section{NORMATIVA}

Presidencia. Decreto I650 del is de julio de I977, sobre el régimen y la administración de los seguros sociales obligatorios.

Presidencia. Decreto 687 de 2014, sobre el acceso de pensionados a los servicios de Cajas de Compensación Familiar.

Presidencia, Decreto $55^{8}$ del 27 de abril de 2020, sobre disminución temporal de la cotización al sistema de pensiones, art. I. https://www.funcionpublica.gov.co/eva/gestornormativo/ norma.php? $\mathrm{i}=\mathrm{I}$ I 3620

Ley i 2 del i6 de enero de 1975, sobre el régimen de pensiones de jubilación, Diario Oficial n. ${ }^{\circ} 34.245$, art. 7 .

Ley 137 del 2 de junio de I994, sobre los estados de excepción.

Ley 1429 del 29 de diciembre de 2010 , sobre formalización y generación de empleo, Diario Oficial n. ${ }^{\circ}$ 47.937. http://www.secretariasenado.gov.co/senado/basedoc/ley_I429_20Io.html

Ministerio del Trabajo, Decreto 4Io8 del 2 de noviembre de 2008, sobre objetivos y estructura del Ministerio de Trabajo. 
Ministerio del Trabajo. Decreto 1072 del 26 de mayo de 2015, Decreto Único Reglamentario del Sector Trabajo.

\section{JURISPRUDENCIA}

Corte Constitucional, Sala Plena, Sentencia C-834 del ro de octubre de 2007, M. P.: Humberto Antonio Sierra Porto. https://www.corteconstitucional.gov.co/relatoria/2007/C-834-07. htm

Corte Constitucional, Sentencia C-428 del I. ${ }^{\circ}$ de julio de 2009, M. P.: Mauricio González Cuervo. https://www.corteconstitucional.gov.co/RELATORIA/2009/C-428-o9.htm

Corte Constitucional, Sala Plena, Sentencia C-372 del I 2 de mayo de 201 I, M. P.: Jose Ignacio Pretelt Chaljub. https://www.corteconstitucional.gov.co/relatoria/20I I/c-372-I I.htm

Corte Constitucional, Sala Plena, Sentencia C-258 del 23 de julio de 2020, M. P.: Antonio José Lizarazo Ocampo. https://www.corteconstitucional.gov.co/Relatoria/2020/C-258-20. htm 